ANNALES

POLONICI MATHEMATICI

$82.1(2003)$

\title{
New variational principle and duality for an abstract semilinear Dirichlet problem
}

\author{
by MAREK GALEWSKI (Łódź)
}

\begin{abstract}
A new variational principle and duality for the problem $L u=\nabla G(u)$ are provided, where $L$ is a positive definite and selfadjoint operator and $\nabla G$ is a continuous gradient mapping such that $G$ satisfies superquadratic growth conditions. The results obtained may be applied to Dirichlet problems for both ordinary and partial differential equations.
\end{abstract}

1. Introduction. We shall prove the existence of solutions to the problem

$$
L x=\nabla G(x),
$$

where $L$ is defined on a separable real Hilbert space $D(L)$ with values in a separable real Hilbert space $Y$ with scalar product $\langle\cdot, \cdot\rangle$. We assume

(A1) $\quad D(L)$ is dense in $Y ; L$ is a selfadjoint and positive definite linear operator, i.e. there is a constant $\alpha>0$ such that for all $x \in D(L)$,

$$
\langle L x, x\rangle \geq \alpha\|x\|^{2} \text {. }
$$

By (1.2) it follows that $R(L)=Y$ and the inverse operator $L^{-1}: Y \rightarrow$ $D(L)$ is continuous and selfadjoint. By the properties of $L$ it follows (see [3]) that there exists (exactly one) operator $S$, called the square root operator, selfadjoint and such that $S^{2}=L$. The domain of $S$, which is a subspace of $Y$, is denoted by $D(S)$. We endow $D(S)$ with the following scalar product:

$$
\left\langle x_{1}, x_{2}\right\rangle_{D(S)}=\left\langle x_{1}, x_{2}\right\rangle+\left\langle S x_{1}, S x_{2}\right\rangle \text {. }
$$

Then the norm in $D(S)$, i.e.

$$
\|x\|_{D(S)}=\sqrt{\|x\|_{Y}^{2}+\|S x\|_{Y}^{2}}
$$

makes it into a complete space. The space $D(S)$ is dense in $Y$ and $D(L)$ is dense in $D(S)$ (see [3], [12]). Moreover $S x \in D(S)$ for any $x \in D(L)$.

2000 Mathematics Subject Classification: 35A15, 70G99.

Key words and phrases: abstract Dirichlet problem, dual variational method, existence of solutions. 
Now we shall state the assumption on the right hand side of the equation considered.

$\nabla G: D(S) \rightarrow D(S)$ is a gradient mapping continuous on $D(S)$ in the topology inherited from $Y$. The potential $G: Y \rightarrow \mathbb{R}$ is lower semicontinuous and convex, $G(0)<\infty$. There exist constants $q \geq q_{1} \geq 2, k_{1}, l_{1}>0, k_{2}, l_{2} \geq 0$ such that for any $x \in Y$,

$\|\nabla G(x)\| \leq k_{1}\|x\|^{q-1}+k_{2}, \quad G(x) \geq l_{1}\|x\|^{q_{1}}+l_{2}$.

The action functional $J: D(S) \rightarrow \mathbb{R}$ is defined by

$$
J(x)=\frac{1}{2}\langle S x, S x\rangle-G(x) .
$$

and the dual functional $J_{D}: D(S) \rightarrow \mathbb{R}$ is given by the formula

$$
J_{D}(p)=G^{*}(S p)-\frac{1}{2}\langle p, p\rangle,
$$

where $G^{*}: Y \rightarrow \mathbb{R}$ denotes the Fenchel-Young conjugate of $G: Y \rightarrow \mathbb{R}$ (see [2]). We observe that both $J$ and $J_{D}$ are unbounded on their respective domains. Hence we have to apply the dual variational method and so we will investigate the following system:

$$
S x=p, \quad S p=\nabla G(x),
$$

where $x \in D(L), p \in D(S)$. Necessary conditions for the existence of a solution to (1.4) are obtained by duality results. The existence is a consequence of a modification of the well known Weierstrass theorem.

The abstract variational principle which we derive enables one to consider linear differential equations of even order. We consider an abstract problem, while in [7]-[11] the duality theory is developed for each Dirichlet problem independently. We use methods of Hilbert spaces which not only make the reasoning clear but also enable us to derive from our results some results known so far. The main difference in comparison to known abstract variational principles [4]-[6] is that the growth assumptions are superquadratic. For the quadratic case see also [1]. Neither the least action principle nor the dual least action principle can be applied here. Hence a duality, based on the duality provided in [8], is derived. The system (1.4), which may be viewed as a system of Hamilton's equations, seems to be new.

We make an additional assumption which is usually satisfied in concrete applications to both O.D.E. and P.D.E.

(A3) $\quad D(S)$ is compactly imbedded in $Y$; for any sequence $\left\{x_{n}\right\}$ with $x_{n} \in D(L)$,

$$
\lim _{n \rightarrow \infty}\left\|x_{n}-\bar{x}\right\|_{D(S)}=0
$$

iff

$$
\lim _{n \rightarrow \infty}\left\|S x_{n}-S \bar{x}\right\|_{Y}=0
$$


a set $A \subset D(L)$ is bounded in $D(S)$ iff there exists a constant $M>0$ such that for any $x \in A$ we have

$$
\|S x\| \leq M \text {. }
$$

2. Duality results. The solution of equation (1.1) is understood in the following sense.

Definition 2.1. We say that $x \in D(L)$ is a solution to equation (1.1) if there exists an element $p \in S(D(L))$ such that system (1.4) is satisfied.

Now we define subsets of $D(L)$ and $D(S)$ on which we will investigate the action and dual action functionals. Put

$$
\widetilde{X}_{k}=\left\{v \in D(L):\|v\|_{Y} \leq k\right\}
$$

where $k>0$ is a number satisfying the inequality

$$
\left\|L^{-1}\right\|\left(k_{1} k^{q-1}+k_{2}\right) \leq k .
$$

Definition 2.2. $X$ is a subset of $\widetilde{X}_{k}$ such that for each $x \in X$ the relation

$$
L \widetilde{x}=\nabla G(x)
$$

implies that $\widetilde{x} \in X$.

LEMma 2.3. There exists a nonempty set $X$ as in Definition 1.2.

Proof. We shall show that $\widetilde{X}_{k}$ has the property from the definition of $X$. Fix $x \in \widetilde{X}_{k}$. Since $L$ is invertible the equation $L \widetilde{x}=\nabla G(x)$ has exactly one solution. By (1.3), definition of $\widetilde{X}_{k}$ and (2.1) we obtain

$$
\|\widetilde{x}\|_{Y} \leq\left\|L^{-1}\right\|\left(k_{1} k^{q-1}+k_{2}\right) \leq k .
$$

Hence $\widetilde{x} \in \widetilde{X}_{k}$. In consequence we may put $X=\widetilde{X}_{k}$.

Now we define a set on which the dual action functional will be considered; we denote it by $X^{d}$.

Definition 2.4. We put

$$
X^{d}=S(X)
$$

Remark 1. Of course, $X^{d} \subset S(D(L))$. The set $X^{d}$ is nonempty since $X$ is. Moreover for each $p \in X^{d}$ there exists exactly one $x \in X$ such that $S x=p$.

Let us define a perturbation $J_{x}: Y \rightarrow \mathbb{R}$ of the functional $J$. Let $x \in X$. For $c \in Y$ put

$$
J_{x}(c)=-\frac{1}{2}\langle S x, S x\rangle+G(x+c) .
$$

Let $p \in X^{d}$. Define a type of conjugate of $J_{x}$ by

$$
J_{x}^{\#}(p)=\sup _{c \in Y}\{\langle c, S p\rangle-G(x+c)\}+\frac{1}{2}\langle S x, S x\rangle .
$$


By the definition and properties of the Fenchel-Young transformation [2], we have

$$
J_{x}^{\#}(p)=G^{*}(S p)+\frac{1}{2}\langle S x, S x\rangle-\langle S p, x\rangle .
$$

In the proof of the duality principle we shall use the lemmas below.

LEMma 2.5. For any $p \in X^{d}$,

$$
\sup _{x \in X}-J_{x}^{\#}(p)=-J_{D}(p) .
$$

Proof. Fix $p \in X^{d}$. Since $S$ is selfadjoint and $X \subset D(L) \subset Y$ we obtain

$$
\begin{aligned}
\sup _{x \in X}\{\langle x, S p\rangle- & \left.\frac{1}{2}\langle S x, S x\rangle\right\}-G^{*}(S p) \\
& \leq \sup _{x \in X}\left\{\langle S x, p\rangle-\frac{1}{2}\langle S x, S x\rangle\right\}-G^{*}(S p) \\
& \leq \sup _{v \in Y}\left\{\langle v, p\rangle-\frac{1}{2}\langle v, v\rangle\right\}-G^{*}(S p)=\frac{1}{2}\langle p, p\rangle-G^{*}(S p) .
\end{aligned}
$$

Hence

$$
\sup _{x \in X}\left\{\langle x, S p\rangle-\frac{1}{2}\langle S x, S x\rangle\right\}-G^{*}(S p) \leq \frac{1}{2}\langle p, p\rangle-G^{*}(S p) .
$$

By Remark 1 it follows that for a given $p \in X^{d}$ there exists $x_{p} \in X$ satisfying $S x_{p}=p$. We then have

$$
\left\langle x_{p}, S p\right\rangle-\frac{1}{2}\left\langle S x_{p}, S x_{p}\right\rangle-G^{*}(S p)=\langle p, p\rangle-\frac{1}{2}\langle p, p\rangle-G^{*}(S p)=\frac{1}{2}\langle p, p\rangle-G^{*}(S p) .
$$

In consequence

$$
\left\langle x_{p}, S p\right\rangle-\frac{1}{2}\left\langle S x_{p}, S x_{p}\right\rangle-G^{*}(S p)=\frac{1}{2}\langle p, p\rangle-G^{*}(S p) .
$$

Hence by (2.2), (2.3) and

$$
\left\langle x_{p}, S p\right\rangle-\frac{1}{2}\left\langle S x_{p}, S x_{p}\right\rangle-G^{*}(S p) \leq \sup _{x \in X}\left\{\langle x, S p\rangle-\frac{1}{2}\langle S x, S x\rangle\right\}-G^{*}(S p)
$$

we conclude that

$$
\sup _{x \in X}-J_{x}^{\#}(p)=-J_{D}(p)
$$

Lemma 2.6. For $x \in X$,

$$
\sup _{p \in X^{d}}-J_{x}^{\#}(p)=-J(x) .
$$

Proof. Fix $x \in X$. By the inclusion $S\left(X^{d}\right) \subset Y$ and by the convexity and lower semicontinuity of $G$ we have

$$
\begin{aligned}
\sup _{p \in X^{d}}\{\langle x, S p\rangle- & \left.G^{*}(S p)\right\}-\frac{1}{2}\langle S x, S x\rangle \\
& \leq \sup _{v \in Y}\left\{\langle x, v\rangle-G^{*}(v)\right\}-\frac{1}{2}\langle S x, S x\rangle \\
& =G^{* *}(x)-\frac{1}{2}\langle S x, S x\rangle=G(x)-\frac{1}{2}\langle S x, S x\rangle=-J(x) .
\end{aligned}
$$


Hence

$$
\sup _{p \in X^{d}}\left\{\langle x, S p\rangle-G^{*}(S p)\right\}-\frac{1}{2}\langle S x, S x\rangle \leq-J(x) .
$$

By definition of $X^{d}$ for a given $x$ there exists $p_{x} \in X^{d}$ such that $S \widetilde{x}=p_{x}$, where $\widetilde{x} \in X$ is such that $L \widetilde{x}=\nabla G(x)$. It follows that

$$
S p_{x}=\nabla G(x)
$$

and by the properties of the Fenchel-Young transformation we have

$$
G(x)+G^{*}\left(S p_{x}\right)=\left\langle x, S p_{x}\right\rangle .
$$

In consequence

$$
\left\langle x, S p_{x}\right\rangle-G^{*}\left(S p_{x}\right)-\frac{1}{2}\langle S x, S x\rangle=G(x)-\frac{1}{2}\langle S x, S x\rangle=-J(x) .
$$

Now the assertion follows from (2.4), (2.5) and

$$
\left\langle x, S p_{x}\right\rangle-G^{*}\left(S p_{x}\right)-\frac{1}{2}\langle S x, S x\rangle \leq \sup _{p \in X^{d}}\left\{\langle x, S p\rangle-G^{*}(S p)\right\}-\frac{1}{2}\langle S x, S x\rangle .
$$

We may now prove the duality principle.

THEOREM 2.7.

$$
\inf _{x \in X} J(x)=\inf _{p \in X^{d}} J_{D}(p) .
$$

Proof. By Lemmas 2.5 and 2.6 we obtain

$$
\begin{aligned}
\inf _{x \in X} J(x) & =-\sup _{x \in X}-J(x)=-\sup _{x \in X} \sup _{p \in X^{d}}-J_{x}^{\#}(p) \\
& =-\sup _{p \in X^{d}} \sup _{x \in X}-J_{x}^{\#}(p)=-\sup _{p \in X^{d}}-J_{D}(p)=\inf _{p \in X^{d}} J_{D}(p) .
\end{aligned}
$$

3. Variational principles. We shall use the results of duality theory to derive the so called variational principle providing necessary conditions for the existence of a solution to equation (1.1).

TheOrem 3.1. Assume that $\bar{x} \in X$ is such that $-\infty<J(\bar{x})=$ $\inf _{x \in X} J(x)<\infty$. Then there exists $\bar{p} \in X^{d}$ such that $S \bar{p} \in \partial J_{\bar{x}}(0)$ and

$$
\inf _{p \in X^{d}} J_{D}(p)=J_{D}(\bar{p})=J(\bar{x})=\inf _{x \in X} J(x) .
$$

Moreover,

$$
\begin{aligned}
J_{\bar{x}}(0)+J_{\bar{x}}^{\#}(\bar{p}) & =0, \\
J_{D}(\bar{p})-J_{\bar{x}}^{\#}(\bar{p}) & =0 .
\end{aligned}
$$

Proof. Observe that for $\bar{x} \in X$ there exists $\bar{p} \in X^{d}$ such that $S \bar{p}=$ $\nabla G(\bar{x})$. This means that $S \bar{p} \in \partial J_{\bar{x}}(0)$. Hence

$$
J_{\bar{x}}(0)+J_{\bar{x}}^{*}(S \bar{p})=0 .
$$


From $J_{\bar{x}}^{*}(S \bar{p})=J_{\bar{x}}^{\#}(\bar{p})$ it follows that $J_{\bar{x}}(0)+J_{\bar{x}}^{\#} p=0$ and in consequence

$$
G(\bar{x})+G^{*}(S \bar{p})-\langle S \bar{p}, \bar{x}\rangle=0 .
$$

From the above and the Fenchel-Young inequality we obtain

$$
\begin{aligned}
-J(\bar{x}) & =-\frac{1}{2}\langle S \bar{x}, S \bar{x}\rangle+G(\bar{x})=-\frac{1}{2}\langle S \bar{x}, S \bar{x}\rangle-G^{*}(S \bar{p})+\langle S \bar{p}, \bar{x}\rangle \\
& =\langle\bar{p}, S \bar{x}\rangle-\frac{1}{2}\langle S \bar{x}, S \bar{x}\rangle-G^{*}(S \bar{p}) \leq \frac{1}{2}\langle\bar{p}, \bar{p}\rangle-G^{*}(S \bar{p})=-J_{D}(\bar{p}) .
\end{aligned}
$$

Hence $J(\bar{x}) \geq J_{D}(\bar{p})$. By Theorem 2.7 it follows that $J(\bar{x}) \leq J_{D}(\bar{p})$. In consequence, $J(\bar{x})=J_{D}(\bar{p})$. Let us observe that $J_{\bar{x}}(0)=-J(\bar{x})=-J_{D}(\bar{p})$. Hence $-J_{D}(\bar{p})+J_{\bar{x}}^{\#}(\bar{p})=0$.

From Theorem 3.1 we derive a corollary which provides necessary conditions for the existence of a solution to system (1.4).

Corollary 3.2. Assume that there exists an $\bar{x} \in X$ such that $-\infty<$ $J(\bar{x})=\inf _{x \in X} J(x)<\infty$. Then there exists $\bar{p} \in X^{d}$ such that the pair $(\bar{x}, \bar{p})$ satisfies

$$
\begin{gathered}
S \bar{x}=\bar{p}, \\
S \bar{p}=\nabla G(\bar{x}) .
\end{gathered}
$$

Proof. Relation (3.4) has been proved in Theorem 3.1. Equality (3.3) follows from (3.2). Indeed, since $J(\bar{x})=J_{D}(\bar{p})$ we have

$$
\frac{1}{2}\langle S \bar{x}, S \bar{x}\rangle-G(\bar{x})=-\frac{1}{2}\langle\bar{p}, \bar{p}\rangle+G^{*}(S \bar{p}) .
$$

Taking the equality

$$
G(\bar{x})+G^{*}(S \bar{p})-\langle S \bar{p}, \bar{x}\rangle=0
$$

into account, we have

$$
\frac{1}{2}\langle S \bar{x}, S \bar{x}\rangle+\frac{1}{2}\langle\bar{p}, \bar{p}\rangle=\langle S \bar{p}, \bar{x}\rangle .
$$

From the above, the properties of the operator $S$ and the definition of the norm in $Y$ it follows that $\bar{p}=S \bar{x}$.

We shall show that the above results with suitable modifications are valid for minimizing sequences. These will be used in the proof of the existence theorem.

Theorem 3.3. Let $\left\{x_{j}\right\}, x_{j} \in X, j \in \mathbb{N}$, be a minimizing sequence for $J$ and assume that

$$
-\infty<\inf _{j \in \mathbb{N}} J\left(x_{j}\right)=a<\infty .
$$

There exists a sequence $\left\{p_{j}\right\}$ minimizing for $J_{D}$ such that $p_{j} \in X^{d}$ and $S p_{j} \in \partial J_{x_{j}}(0)$ for $j \in \mathbb{N}$. Furthermore,

$$
\inf _{p \in X^{d}} J_{D}(p)=\inf _{j \in \mathbb{N}} J_{D}\left(p_{j}\right)=\inf _{x \in X} J(x)=\inf _{j \in \mathbb{N}} J\left(x_{j}\right)
$$


and for all $j \in \mathbb{N}$ we have

$$
J_{x_{j}}(0)+J_{x_{j}}^{\#}\left(p_{j}\right)=0 .
$$

Moreover, for any $\varepsilon>0$ there exists $j_{0}$ such that for all $j \geq j_{0}$,

$$
J\left(x_{j}\right)-J_{D}\left(p_{j}\right)<\varepsilon .
$$

Proof. Since $x_{j} \in X$ for $j \in \mathbb{N}$, it follows that there exists $p_{j} \in X^{d}$ such that

$$
S p_{j}=\nabla G\left(x_{j}\right)
$$

We will show that $\left\{p_{j}\right\}$ is a minimizing sequence for $J_{D}$. By (3.7) and the Fenchel-Young inequality, for any $j \in \mathbb{N}$ we have

$$
\begin{aligned}
-J\left(x_{j}\right) & =-\frac{1}{2}\left\langle S x_{j}, S x_{j}\right\rangle+G\left(x_{j}\right)=-\frac{1}{2}\left\langle S x_{j}, S x_{j}\right\rangle-G^{*}\left(S p_{j}\right)+\left\langle S p_{j}, x_{j}\right\rangle \\
& =\left\langle p_{j}, S x_{j}\right\rangle-\frac{1}{2}\left\langle S x_{j}, S x_{j}\right\rangle-G^{*}\left(S p_{j}\right) \\
& \leq \frac{1}{2}\left\langle p_{j}, p_{j}\right\rangle-G^{*}\left(S p_{j}\right)=-J_{D}\left(p_{j}\right) .
\end{aligned}
$$

Hence

$$
J\left(x_{j}\right) \geq J_{D}\left(p_{j}\right) .
$$

Take arbitrary $\varepsilon>0$. Observe that since

$$
-\infty<\inf _{j \in \mathbb{N}} J\left(x_{j}\right)=a<\infty,
$$

there exists $j_{0}$ such that $J\left(x_{j}\right)<a+\varepsilon$ for all $j \geq j_{0}$. By (3.8) it follows that $J_{D}\left(p_{j}\right)<a+\varepsilon$ for $j \geq j_{0}$. Now from Theorem 2.7 we deduce that $\inf _{j \in \mathbb{N}} J_{D}\left(p_{j}\right)=a$. In consequence, $\left\{p_{j}\right\}$ is a minimizing sequence for $J_{D}$.

Relation (3.5) follows from (3.7) by using similar arguments to those in the proof of Theorem 3.1 (cf. (3.1)).

Relation (3.6) follows from Theorem 2.7. Indeed, for each $\varepsilon>0$ there exists $j_{0}$ such that for all $j \geq j_{0}$,

$$
J\left(x_{j}\right)<a+\varepsilon=\inf _{l \in \mathbb{N}} J\left(x_{l}\right)+\varepsilon=\inf _{l \in \mathbb{N}} J_{D}\left(p_{l}\right)+\varepsilon \leq J_{D}\left(p_{j}\right)+\varepsilon .
$$

Corollary 3.4. Let $\left\{x_{j}\right\}, x_{j} \in X, j \in \mathbb{N}$, be a minimizing sequence for $J$ and assume that

$$
-\infty<\inf _{j \in \mathbb{N}} J\left(x_{j}\right)=a<\infty .
$$

If $p_{j} \in X^{d}$ and $S p_{j}=\nabla G\left(x_{j}\right)$, then $\left\{p_{j}\right\}$ is a minimizing sequence for $J_{D}$. For all $j \in \mathbb{N}$,

$$
J_{x_{j}}(0)+J_{x_{j}}^{\#}\left(p_{j}\right)=0 .
$$

Moreover, for every $\varepsilon>0$ there exists $j_{0}$ such that for all $j \geq j_{0}$ we have

$$
J\left(x_{j}\right)-J_{D}\left(p_{j}\right)<\varepsilon .
$$


4. Existence of solutions. We shall show that there exists an element $\bar{x} \in D(L)$ and an element $\bar{p} \in S(D(L))$ such that the system (1.4) is satisfied. We will make use of the lemma below.

Lemma 4.1. There exist constants $d_{1}>0, d_{2}$ such that for any $x \in Y$,

$$
G(x) \leq d_{1}\|x\|^{q}+d_{2} .
$$

Proof. Putting $d_{1}=k_{1}+k_{2}$ and $d_{2}=k_{2}+G(0)$, by convexity and Gateaux differentiability of $G$ and by the Schwarz inequality and (1.3) we obtain (4.1).

Theorem 4.2. There exists a pair $(\bar{x}, \bar{p}) \in D(L) \times S(D(L))$ such that

$$
\begin{gathered}
S \bar{x}=\bar{p}, \\
S \bar{p}=\nabla G(\bar{x}) .
\end{gathered}
$$

Proof. We first show that the functional $J$ is bounded from below on $X$. By Lemma 4.1 and the definition of $X$ it follows that there exists a constant $c>0$ such that $G(x) \leq c$ for all $x \in X$. From this and the definition of $J$ we obtain, for any $x \in X$,

$$
J(x)=\frac{1}{2}\langle S x, S x\rangle-G(x) \geq \frac{1}{2}\langle S x, S x\rangle-c=\frac{1}{2}\|S x\|^{2}-c .
$$

Since $X \neq \emptyset$, there exists $b \in \mathbb{R}$ such that the set $S_{b}=\{x \in X: J(x) \leq b\}$ is nonempty. Since $X \subset D(L)$, we deduce from (4.4) and assumption (A3) that $S_{b}$ is bounded in $D(S)$. In consequence, it is relatively weakly compact in $D(S)$. Hence we may find in $S_{b}$ a minimizing sequence $\left\{x_{j}\right\}$ weakly convergent in $D(S)$. By (A3) it follows that $\left\{x_{j}\right\}$ converges strongly in $Y$. We denote its limit by $\bar{x} \in D(S)$.

We shall show that $J(\bar{x})=\inf _{x \in X} J(x)$.

Observe that $J$ is weakly lower semicontinuous on $D(S)$. Indeed, the functional

$$
D(S) \ni x \mapsto \frac{1}{2}\langle S x, S x\rangle \in \mathbb{R}
$$

is convex and lower semicontinuous. Hence it is weakly lower semicontinuous on $D(S)$. Since $S_{b}$ is bounded there exists an open set $A \subset Y$ bounded in $Y$ and containing $S_{b}$. By Lemma 4.1 it follows that $G$ is bounded from above on $A$. Since $G$ is convex it is continuous [2], i.e. $\lim _{n \rightarrow \infty} G\left(x_{n}\right)=G(\bar{x})$. In consequence,

$$
\liminf _{n \rightarrow \infty} J\left(x_{n}\right) \geq J(\bar{x}) .
$$

Then

$$
J(\bar{x})=\inf _{x \in X} J(x) .
$$

We will show that there exists a $\bar{p} \in S(D(L))$ such that $(\bar{x}, \bar{p})$ satisfies system (4.2)-(4.3). We will use the properties of a minimizing sequence for the dual functional. By the definition of $X^{d}$ we can find a sequence $\left\{p_{j}\right\} \subset$ 
$X^{d}$ such that $S p_{j}=\nabla G\left(x_{j}\right)$. By Corollary 3.4 it follows that $\left\{p_{j}\right\}$ is a minimizing sequence for $J_{D}$.

We will show that $\left\{p_{j}\right\}$ converges strongly in $D(S)$. Indeed, since $\left\{x_{j}\right\}$ is strongly convergent in $Y$ and $\nabla G$ is continuous on $D(S)$ (in the topology inherited from $Y$ ), it follows that $\left\{\nabla G\left(x_{j}\right)\right\}$ converges to $\nabla G(\bar{x})$. By the above and continuity of $S^{-1}$ we see that the sequence

$$
p_{j}=S^{-1} \nabla G\left(x_{j}\right) \in D(S)
$$

is strongly convergent. We denote its limit by $\bar{p} \in D(S)$. Hence

$$
\bar{p}=S^{-1} \nabla G(\bar{x})
$$

proving (4.3).

We now show that $(\bar{p}, \bar{x})$ also satisfies (4.2). By Corollary 3.4 there exists a numerical sequence $\left\{\varepsilon_{n}\right\}, \varepsilon_{n}>0, \varepsilon_{n} \rightarrow 0$, having the property: for each $n$ there exists $j_{n}$ such that for all $j \geq j_{n}$,

$$
\frac{1}{2}\left\langle S x_{j}, S x_{j}\right\rangle+\frac{1}{2}\left\langle p_{j}, p_{j}\right\rangle-\left\langle x_{j}, S p_{j}\right\rangle \leq \varepsilon_{n} .
$$

We may assume that $j_{n} \rightarrow \infty$. Since $\left\{x_{j}\right\}$ is strongly convergent in $Y$ and $\left\{S p_{j}\right\}$ converges strongly to $\nabla G(\bar{x})$ in $Y$, it follows that $\lim _{j \rightarrow \infty}\left\langle x_{j}, S p_{j}\right\rangle=$ $\langle\bar{x}, \nabla G(\bar{x})\rangle$. Taking $n \rightarrow \infty$ we obtain

$$
\begin{aligned}
0 & \geq \liminf _{j \rightarrow \infty}\left(\frac{1}{2}\left\langle S x_{j}, S x_{j}\right\rangle+\frac{1}{2}\left\langle p_{j}, p_{j}\right\rangle-\left\langle x_{j}, S p_{j}\right\rangle\right) \\
& \geq \liminf _{j \rightarrow \infty} \frac{1}{2}\left\langle S x_{j}, S x_{j}\right\rangle+\lim _{j \rightarrow \infty} \frac{1}{2}\left\langle p_{j}, p_{j}\right\rangle-\lim _{j \rightarrow \infty}\left\langle x_{j}, S p_{j}\right\rangle \\
& \geq \frac{1}{2}\langle S \bar{x}, S \bar{x}\rangle+\frac{1}{2}\langle\bar{p}, \bar{p}\rangle-\langle\bar{x}, \nabla G(\bar{x})\rangle .
\end{aligned}
$$

From the above, relation (4.3) and the Fenchel-Young inequality we obtain

$$
0 \geq \frac{1}{2}\langle S \bar{x}, S \bar{x}\rangle+\frac{1}{2}\langle\bar{p}, \bar{p}\rangle-\langle\bar{x}, S \bar{p}\rangle \geq 0 .
$$

The definition of the norm in $Y$ now yields (4.2). The limit $\bar{x}$ of $\left\{x_{j}\right\}$ belongs to $D(S)$. From (4.2) and (4.3) we obtain

$$
S^{2} \bar{x}=\nabla G(\bar{x}) .
$$

Since $S^{2}=L$, we conclude that $\bar{x} \in D(L)$. In consequence, $\bar{p} \in S(D(L))$.

\section{References}

[1] H. Amann, On the unique solvability of semilinear operator equations in Hilbert spaces, J. Math. Pures Appl. 61 (1982), 149-175.

[2] I. Ekeland and R. Temam, Convex Analysis and Variational Problems, NorthHolland, Amsterdam, 1976.

[3] T. Kato, Perturbation Theory for Linear Operators, Springer, 1980.

[4] J. Mawhin, Problèmes de Dirichlet variationnels non linéaires, Presses Univ. Montréal, 1987. 
[5] J. Mawhin and A. Fonda, Iterative and variational methods for the solvability of some semilinear equations in Hilbert spaces, J. Differential Equations 98 (1992), $355-375$.

[6] J. Mawhin and M. Willem, Critical Point Theory, Springer, New York, 1989.

[7] A. Nowakowski, A new variational principle and duality for periodic solutions of Hamilton's equations, J. Differential Equations 97 (1992), 174-188.

[8] A. Nowakowski and A. Rogowski, On the new variational principles and duality for periodic solutions of Lagrange equations with superlinear nonlinearities, J. Math. Anal. Appl. 264 (2001), 168-181.

[9] - - - Two-point boundary value problem with superlinear nonlinearities, Nonlinear Anal. 47 (2001), 67-77.

[10] —, - Multiple positive solutions for a nonlinear Dirichlet problem with nonconvex vector valued response, to appear.

[11] A. Orpel, Duality and variational principle of the Dirichlet problem with superlinear nonlinearities, Faculty of Math., Łódź Univ., 2000, preprint no. 02.

[12] K. Yosida, Functional Analysis, Springer, New York, 1974.

Faculty of Mathematics

University of Łódź

Banacha 22

90-238 Łódź, Poland

E-mail: galewski@imul.uni.lodz.pl

Reçu par la Rédaction le 28.5.2002

Révisé le 3.7.2003 